\title{
Assessment of the variability in the dimensions of the intact pelvic canal in South Africans: a pilot study
}

\author{
S. Jagesur ${ }^{a}, *$, A. Wiid ${ }^{a}$, S. Pretorius ${ }^{b}$, M.C. Bosman ${ }^{a}$, A.C. Oettlé ${ }^{a}$ \\ ${ }^{a}$ Department of Anatomy, School of Medicine, Faculty of Health Sciences, University of Pretoria, Private Bag x323, \\ Arcadia, Pretoria, 0007, Republic of South Africa. \\ ${ }^{b}$ Department of Actuarial Science. University of Pretoria, Pretoria, 0001, Republic of South Africa.
}

Abbreviated title: Pelvic canal in South Africans

*Corresponding author. Ms. Suvasha Jagesur (MSc), tel: +27 736988 701, E-mail address:

suvasha.jagesur@gmail.com

\begin{abstract}
Cephalopelvic disproportion is common among Africans and is a major cause of maternal and perinatal mortality and morbidity. As the dimensions of the pelvis may vary between populations and according to stature and age, they need to be considered during childbirth and also in the planning and performance of pelvic and perineal procedures. The aim of this study was to assess the possible variations in the dimensions of the intact pelvic canal in South Africans and their implications. Eighty intact cadaver pelves, belonging to 40 white South Africans (20 males and 20 females) and 40 black South Africans (20 males and 20 females) were used for both metric and geometric morphometric analyses. Pelvic inlet shapes did not differ significantly between groups but pelvic inlet and midpelvic dimensions were the greatest in whites and females. The pubic symphyseal length was the greatest in whites and males and the smallest in black females, resulting in a smaller pelvic cavity anteriorly than for white females. Pelvic outlet shapes varied significantly between sexes in white South Africans and between populations in males. Females presented with the greatest dimensions. Black females presented with an elongated anteroposterior outlet diameter. Certain transverse pelvic diameters correlated positively with age in white males and with height in females. In planning childbirth options, the smaller pelvic inlet of black females and stature-dependent diameters should be considered. Pelvic and perineal
\end{abstract}


surgery may be technically more challenging because of smaller pelvic dimensions in black South Africans, especially in males.

Keywords: Pelvic inlet; Midpelvis; Pelvic outlet; Shape analysis; Pelvic canal

\section{Introduction}

Measurable differences in the size and shape of the skeletal components of the pelvis between groups, including black and white South Africans, have been documented in the literature (Loth and Henneberg, 1996; Patriquin et al., 2002, 2003, 2005). Apart from the variations noted between population groups, other factors such as stature and aging may also have an influence on pelvic skeletal dimensions (İşcan, 2005; İşcan and Steyn, 2013). These variations in pelvic dimensions among South Africa's population groups and with stature and aging considered become important when decisions regarding method of parturition are made or pelvic procedures are planned. Stature, for instance, is often used as an early warning for possible cephalopelvic disproportion: the disproportion of the foetal head size as compared to the size of the maternal pelvis during delivery. Cephalopelvic disproportion is not an uncommon finding in Africans and could be a major cause of maternal and perinatal mortality and morbidity as indicated by Leong (2006). Constrictive pelvic dimensions could not only have far-reaching implications in obstetrics practice, but could also play a role in surgical procedures involving male and female pelvic structures.

The classic dimensions that are usually of importance are the diameters of the pelvic inlet (anteroposterior and transverse), midpelvis (interspinous distance) and pelvic outlet (anteroposterior and transverse). Several authors have related the size of the anteroposterior inlet diameter to the height of the woman and the obstetric outcome (Adadevoh et al., 1989; Bernard 1952; Merchant et al., 2001; Stewart et al., 1979). The interspinous distance is also important to consider in parturition, as it is normally the narrowest part of the pelvic canal through which the foetal head must pass during birth. A narrow pelvic outlet may further predispose a woman to a difficult vaginal delivery. A rapid assessment of the outlet adequacy may be made by estimating the angle of the subpubic arch (Frudinger et al., 2002). The smaller the angle, the closer together the ischial tuberosities are and, therefore, the narrower the pelvic outlet. 
Specific dimensions of the bony pelvis - relating to pelvic procedures in both males and females and during childbirth - have not been investigated in South Africans. It is therefore of value to study the possible variations in the dimensions of the pelvis between population groups, as well as their correlation with stature and aging. These diameters and their relationships will be helpful in assessing the possibility of a favourable outcome in vaginal deliveries. They will also be useful when surgical procedures in both sexes are planned, as a small pelvic canal, for example, may impede vision, access and space for surgical excision (Hong et al., 2007; Killeen et al., 2010; Salerno et al., 2007).

The purpose of this study was to assess certain clinically relevant dimensions on defleshed, but intact South African cadaver pelves and to take note of variations between populations and sexes and the effects of short stature and aging. The possible implications of the variations between groups are considered in the discussion section.

\section{Materials and methods}

A total of 80 intact cadaver pelves from the anatomy departments of both the University of Pretoria and the former Medunsa Campus, University of Limpopo now called "Sefako Makgatho Health Sciences University" (SMU) were sampled. Cadavers used in this study were obtained between 2005 and 2009 as unclaimed or donated bodies from the surrounding hospitals. The sample group was evenly distributed between the sexes and population groups (black and white South Africans). The population group and sex of all individuals were known. Seventy-two of the 80 individuals had known ages (Table 1). Pelves that exhibited pathological features were excluded.

Stature was derived by regression analysis using left femur length measurements. Lundy and Feldesman (1987) developed regression formulae incorporating femur lengths for the estimation of antemortem stature in male and female black South Africans, whilst Dayal et al. (2008) similarly developed formulae for male and female white South Africans. The living stature was then determined by adding Raxter's value for soft tissue (Bidmos and Manger, 2012).

Femur length was measured on an osteometric board using the technique described by MooreJanson et al. (1994). The maximum length of the femur is defined as the distance from the most superior point on the head of the femur to the most inferior point on the distal condyles. The posterior surface of the femur was placed parallel to the long axis of the osteometric board. The 
Table 1. Variables of intact cadaver pelves within sex-population group. Group means, standard deviations (SD), and significant p-values in black males (N=20), white males $(\mathrm{N}=20)$, white females $(\mathrm{N}=20)$ and black females $(\mathrm{N}=20)$ for the variables used in this study. Measurements are in mm, subpubic angle in degrees, stature in cm and age in years.

\begin{tabular}{|c|c|c|c|c|c|c|c|c|}
\hline \multirow{2}{*}{ Variable } & \multicolumn{2}{|c|}{ Black SA male } & \multicolumn{2}{|c|}{ White SA male } & \multicolumn{2}{|c|}{ Black SA female } & \multicolumn{2}{|c|}{ White SA female } \\
\hline & mean & SD & mean & SD & mean & SD & mean & SD \\
\hline AB: Pelvic inlet: anteroposterior & $113.57^{\mathrm{SP} *}$ & 10.30 & $123.44^{\mathrm{P*1}}$ & 11.32 & $119.56^{\mathrm{SP}}$ & 7.10 & $128.62^{P}$ & 11.94 \\
\hline $\mathrm{C}_{\mathrm{L}} \mathrm{C}_{\mathrm{R}}$ : Pelvic inlet: transverse & $112.04^{\mathrm{SP} *}$ & 10.22 & $126.54^{\mathrm{P} * 2}$ & 8.69 & $118.88^{\mathrm{SP*1}}$ & 7.74 & $130.76^{\mathrm{P}^{*}}$ & 7.65 \\
\hline BD: Length of pubic symphysis & $44.23^{\mathrm{SP} *}$ & 5.40 & $48.63^{\mathrm{P*}}$ & 4.98 & $40.04^{\mathrm{SP}}$ & 6.13 & $45.31^{\mathrm{P} 1}$ & 5.54 \\
\hline $\mathrm{F}_{\mathrm{L}} \mathrm{F}_{\mathrm{R}}:$ Midpelvis: interspinous & $78.84^{\mathrm{S} * \mathrm{P} *}$ & 9.23 & $90.03^{\mathrm{S} \mathrm{P}^{*}}$ & 6.81 & $98.93^{\mathrm{S}^{*}}$ & 7.82 & $102.66^{\mathrm{S}^{*}}$ & 10.90 \\
\hline $\mathrm{DE}_{\mathrm{L}}:$ Ischiopubic ramus & $74.82^{\mathrm{SP}}$ & 5.36 & $79.30^{\mathrm{P} 2}$ & 6.17 & $80.42^{\mathrm{S} 1}$ & 9.82 & $80.06^{1}$ & 6.54 \\
\hline $\mathrm{E}_{\mathrm{L}} \mathrm{E}_{\mathrm{R}}:$ Pelvic outlet: intertuberous & $83.45^{\mathrm{S} \mathrm{P}^{*}}$ & 8.31 & $98.34^{\mathrm{S} \mathrm{P}^{*}}$ & 10.00 & $110.19^{S^{*}}$ & 9.06 & $109.96^{\mathrm{S}^{* 1}}$ & 12.32 \\
\hline DG: Pelvic outlet: anteroposterior & $92.03^{\mathrm{S}}$ & 8.63 & 95.00 & 8.78 & $99.60^{\mathrm{S}}$ & 11.15 & 94.87 & 12.92 \\
\hline Subpubic angle & $69.09^{\mathrm{S} * \mathrm{P}}$ & 7.90 & $74.07^{\mathrm{S} * \mathrm{P}}$ & 7.31 & $85.49^{S^{*}}$ & 7.50 & $86.85^{\mathrm{S}^{*}}$ & 7.97 \\
\hline Stature & 169.63 & 8.15 & 175.36 & 8.47 & $158.27^{\mathrm{S}}$ & 7.18 & 164.31 & 6.04 \\
\hline Age & 47.94 & 15.88 & 68.45 & 15.99 & $40.31^{\mathrm{S}}$ & 18.57 & 69.45 & 17.67 \\
\hline \multicolumn{9}{|l|}{$\mathrm{SA}=$ South Africans } \\
\hline \multicolumn{9}{|c|}{${ }^{1}$ Statistically significant correlation with stature } \\
\hline \multicolumn{9}{|c|}{${ }^{2}$ Statistically significant correlation with age } \\
\hline \multicolumn{9}{|c|}{${ }^{\mathrm{S} / \mathrm{P}}:$ Statistically significant correlation between sex/population group: $0.01<\mathrm{p}<0.05$} \\
\hline $\mathrm{S}^{* / \mathrm{P}^{*}}:$ Statistically significant correla & tween $\operatorname{sex} / \mathrm{p}$ & lation $\mathrm{g}$ & $: p \leq 0.01$ & & & & & \\
\hline
\end{tabular}


medial and lateral condyles were pressed against the vertical end board while applying the movable upright to the femoral head until the maximum length was obtained (Moore-Jansen et al., 1994).

The pelves were defleshed and stripped to the bone without disarticulation to facilitate the identification of bony landmarks and the measurement of the distances between them. Measurements were taken from bone to bone but did incorporate joint attachments such as cartilage and ligaments, which would reflect the natural situation and would be more accurate when comparisons with clincial studies were made. Care was taken to keep the specimens moist with embalmimg fluid and covered by linen and waterproofed sheets to prevent dessication of the ligaments and cartilage joints.

The following ten points were marked with pins and then digitised by using a 3DXL MicroScribe ${ }^{\circledR}$ digitiser on intact pelves (Figs. 1 and 2): A: midpoint of the sacral promontory; B: most superior point in the midline of the pubic symphysis; $C_{L}$ and $C_{R}$ : left and right widest points on the pelvic inlet; $\mathrm{D}$ : most inferior point in the midline of the pubic symphysis; $\mathrm{E}_{\mathrm{L}}$ : most inferior point on the ischial tuberosity (left); $\mathrm{E}_{\mathrm{R}}$ : most inferior point on the ischial tuberosity (right); $F_{L}$ : ischial spine (left); $F_{R}$ : ischial spine (right); G: lowest limit of coccyx.

Figure 1. Anteroinferior view of landmarks on wet intact pelves

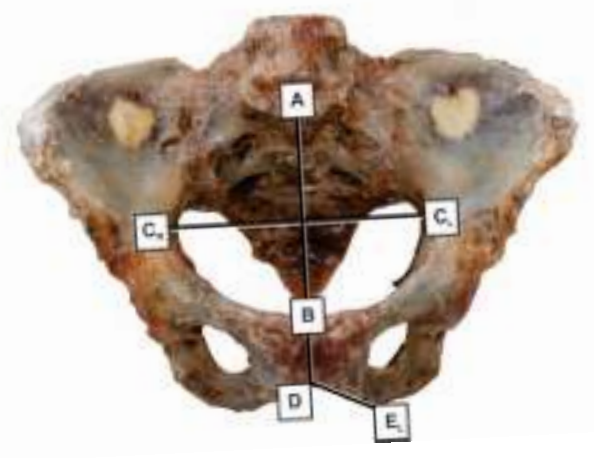

Figure 2. Inferior view of landmarks on wet intact pelves 


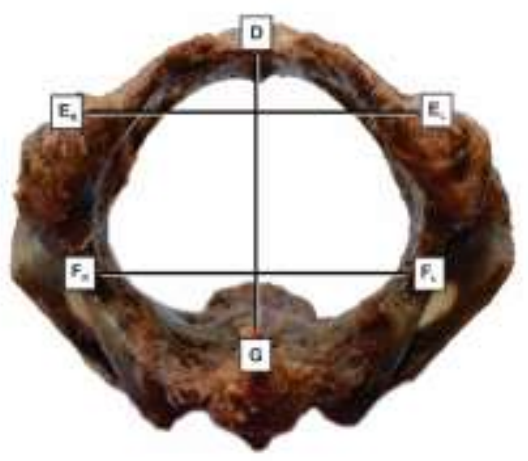

Distances and angles were calculated using standard mathematical techniques, based on the points measured on a three dimensional Cartesian coordinate system. The following distances were calculated between the various landmarks: AB: pelvic inlet antero-posterior (AP) diameter; $\mathrm{C}_{\mathrm{L}} \mathrm{C}_{\mathrm{R}}$ : pelvic inlet transverse diameter; $\mathrm{BD}$ : length of pubic symphysis; $\mathrm{DE} \mathrm{L}_{\mathrm{L}}$ : distance from the most inferior point in the midline of the pubic symphysis to the most inferior point on the left ischial tuberosity (length of the ischiopubic ramus); $\mathrm{E}_{\mathrm{L}} \mathrm{E}_{\mathrm{R}}$ : intertuberous (transverse outlet) diameter; $\mathrm{F}_{\mathrm{L}} \mathrm{F}_{\mathrm{R}}$ : interspinous (midpelvis) diameter; $\mathrm{DG}$ : pelvic outlet $\mathrm{AP}$ diameter and the subpubic angle between points $\mathrm{E}_{\mathrm{L}}, \mathrm{E}_{\mathrm{R}}$ and $\mathrm{D}$.

Basic descriptive statistics of all the data were performed; i.e. the mean and standard deviation. Statistical comparisons of parameters were made between the various sex-population groups as well as linear correlations to stature and age using ANOVA. Inter-observer errors were tested on a later occasion by another observer familiar with the method. Thirty randomly selected intact pelves were remeasured, using the reliability coefficient.

The digitised landmarks were further considered for shape analyses. The landmarks were grouped into those describing the pelvic inlet landmarks (Fig. 3), pelvic outlet landmarks including ischial spines (Fig. 4) and pelvic canal landmarks (which included those of the pelvic inlet, midpelvis and outlet) (Fig. 5 and Fig. 6). Shape analyses were performed on all the intact pelves by introducing the data into Morphologika2 v2.5. A generalised full procrustes analysis was performed to correct for orientation and size differences. The distribution of variance was plotted for principal components 1 and 2 representing the main shape differences to visually evaluate the scattering patterns amongst the groups. Two group multivariate permutation test of PAST, PAleontologicalSTatistics v1.92 was used to test for statistical significant differences between groups (Hammer et al., 2001). 
Figure 3. Landmarks used in shape analysis for the pelvic inlet

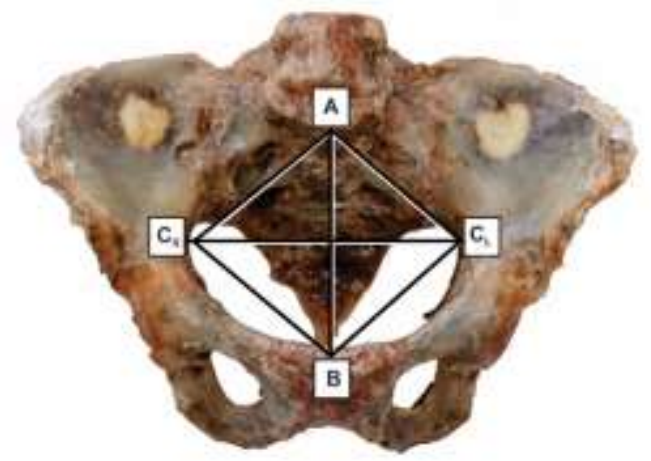

Figure 4. Landmarks used in shape analysis for the pelvic outlet and ischial spines

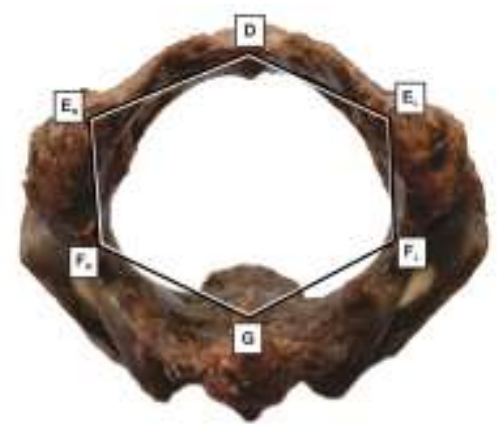

Figure 5. Landmarks used in shape analysis for the pelvic canal (seen from anteroinferiorly)

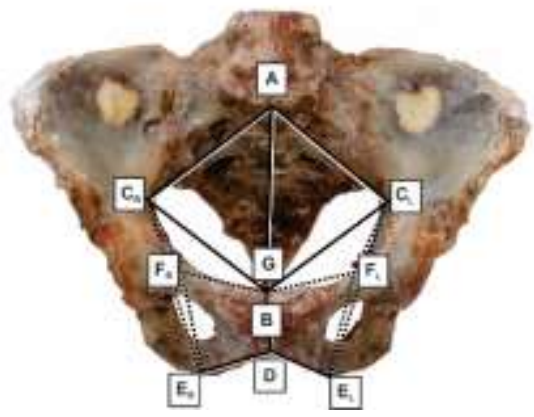


Figure 6. Landmarks used in shape analysis for the pelvic canal (seen from lateral)

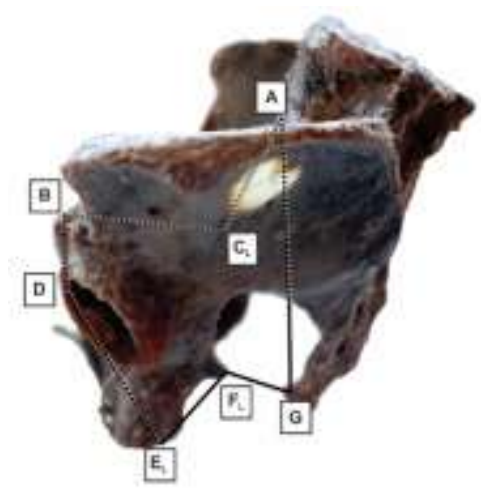

\section{Ethical Considerations}

The skeletal material used in this study was obtained between 2005 and 2009 as unclaimed or donated bodies from the surrounding hospitals under the National Health Act No. 61 of 2003. Ethical clearance was granted by the Faculty of Health Sciences Research Ethics Committee, University of Pretoria, under the Protocol Number S42/2011.

\section{Results}

The basic descriptive statistics of the measured distances are given in Table 1 and principal component plots in figures 7 and 8 . The pelvic inlet shape, defined by the position of the four landmarks as described before, did not differ statistically between groups (sexes: Fig 7a and population groups: Fig. 7b). In contrast pelvic inlet dimensions were statistically significantly greater in females compared to males when considering the total sample and black South Africans, but not significantly in white South Africans (Table 1). When comparing population groups within sexes, statistically significantly greater dimensions were found in white South Africans than in black South Africans. When comparing females, the pelvic inlet transverse diameter $\left(C_{L} C_{R}\right)$ differed at a lower level of significance than the antero-posterior diameter $(A B)$, which could be indicative of some variation in the inlet shape between the population groups (Table 1). In other words, the greater pelvic inlet dimensions noted in South African white vs. South African blacks especially in females seemed to have been associated with relatively wider pelvic inlet shapes. 
When comparing pelvic outlet shape between sexes defined by the position of the six landmarks as described before, statistically significant variations were noted in the total sample $(\mathrm{p}=0.0005)($ Fig. 8a) and when considering white South Africans in isolation $(\mathrm{p}=0.0145)$ (Fig. $8 b)$. Sex variations in the pelvic outlet shape were only statistical significant at a level of $10 \%$ when considering black South Africans ( $\mathrm{p}=0.0985)$. In females the urogenital triangle, as defined by the distance on either side between the pubic symphysis to the ischial tuberosity and the distance between the ischial tuberosities, was relatively larger and therefore directed further and more posteriorly as compared to males. The coccyx was directed more posteriorly. These shape differences were also reflected in the metric dimensions.

Figure 7. Principal component 1 versus 2: Pelvic inlet shape comparisons

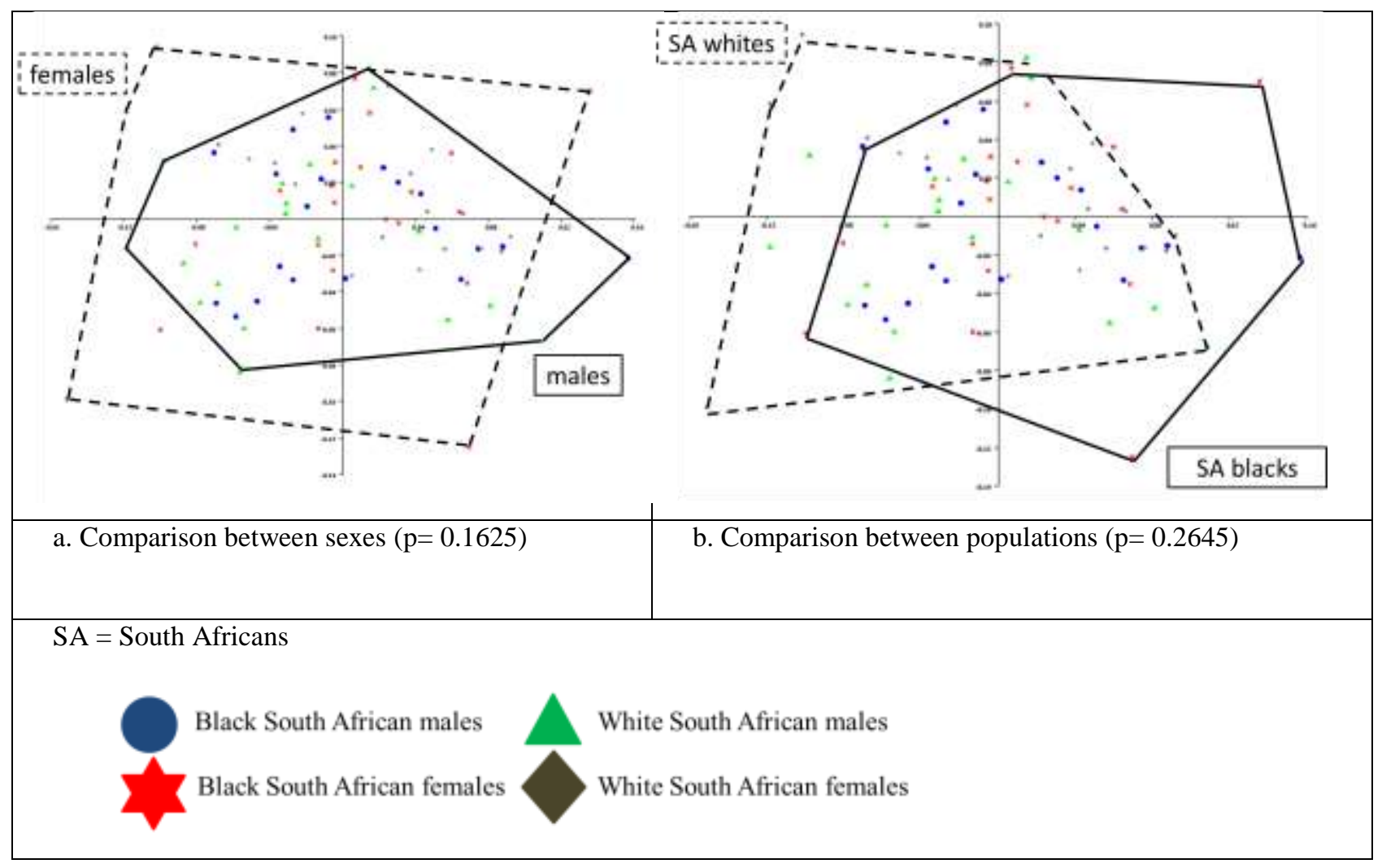


Figure 8. Principal component 1 versus 2: Pelvic outlet comparisons

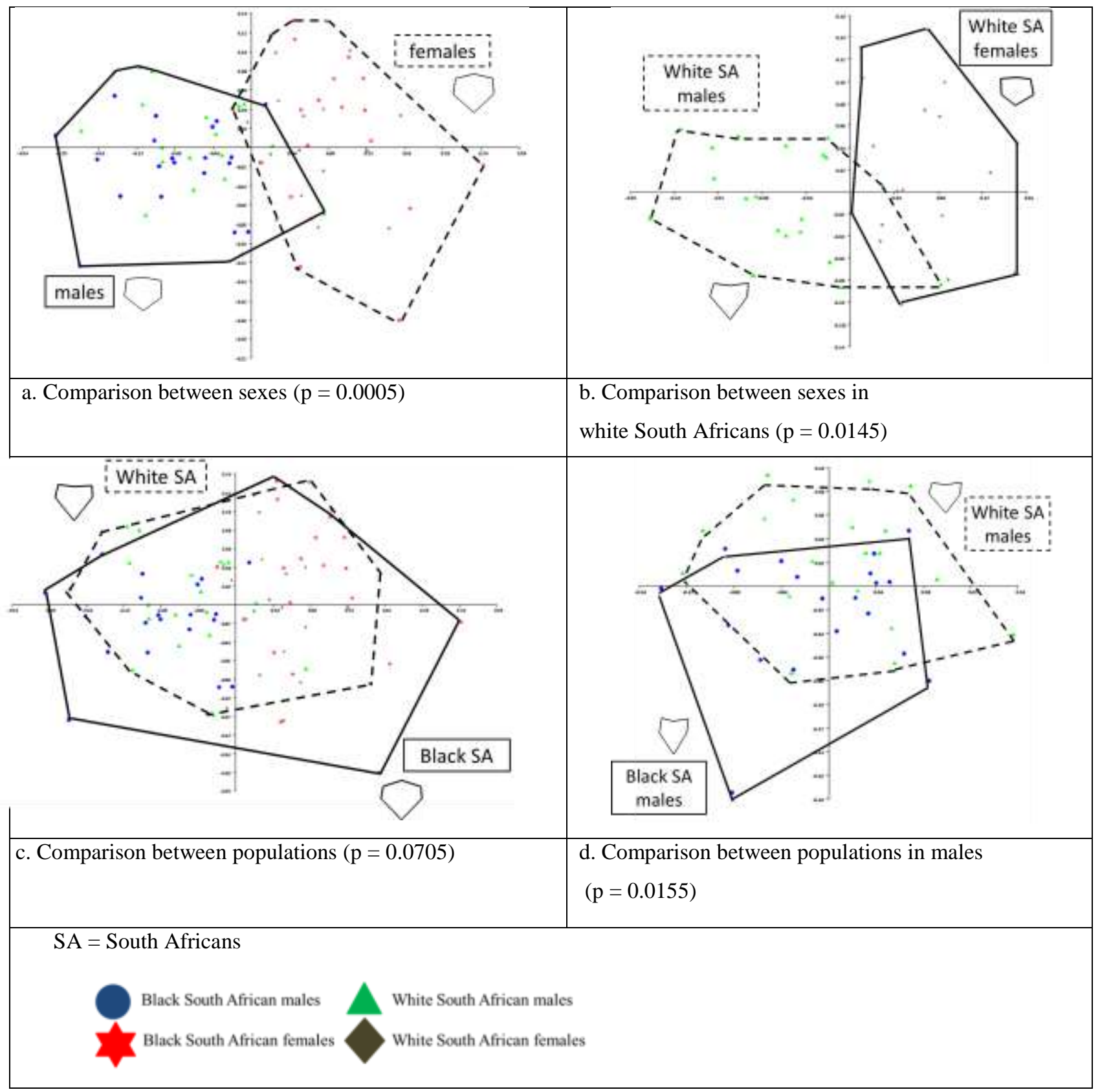

The midpelvic $\left(\mathrm{F}_{\mathrm{L}} \mathrm{F}_{\mathrm{R}}\right)$ dimension/interspinous distance was statistically significantly greater in females compared to males in each population group in isolation: black South Africans ( $\mathrm{p}=$ $0.0000)$ and white South Africans $(\mathrm{p}=0.0005)$. Pelvic outlet dimensions (intertuberous distance $\left(E_{L} E_{R}\right)$, anteroposterior outlet distance $(D G)$, subpubic angle and ischiopubic ramus length $\left(D_{L}\right)$ were the greatest in black South African females and differed significantly from black South African males, who presented with the smallest dimensions. The intertuberous distance and 
along with that the subpubic angle differed the most significantly between the sexes in black South Africans. The intertuberous distance and the subpubic angle also differed statistically significantly between the sexes in white South Africans.

The limitation of the statistically significant intersex differences to the anterior pelvic dimensions in white South Africans could be in keeping with the lower level of statistically significant sex differences in the pelvic outlet shapes in white South Africans as compared to black South Africans. In black South African females the pelvic outlet dimensions were more uniformly increased as compared to males. White South African females presented with wider midpelvic- and pelvic-outlet shapes as compared to white South African males, while all the pelvic outlet dimensions were greater in black South African females as compared to black South African males.

Pelvic outlet shape comparisons between population groups were statistically significant at a level of $10 \%(\mathrm{p}=0.0705)$ in the complete sample (Fig 8c) but statistically significant at a level of $5 \%$ when comparing males: $\mathrm{p}=0.0155$ (Fig 8d). These pelvic outlet shape variations were reflected in the statistically significant greater midpelvic- and transverse pelvic-outlet dimensions (intertuberous $\left(E_{L} E_{R}\right)$, subpubic angle and ischiopubic ramus length $\left(D_{L}\right)$ ) in white South African males as compared to black South African males but not in the anteroposterior outlet distance (DG) distance. Differences between females were not statistically significant.

All sex and population group comparisons involving the pelvic canal shape, defined before as the combined pelvic inlet, midpelvis and outlet shapes, were statistically significant: males compared to females: $\mathrm{p}=0.0005$; black males compared to black females: $\mathrm{p}=0.0005$; black females compared to white females: $\mathrm{p}=0.071$; black males compared to white males: $\mathrm{p}=0.008$; white males compared to white females: $p=0.0005$; black South Africans vs. white South Africans: $\mathrm{p}=0.0415$.

The distance between the pelvic inlet and outlet is reflected in the length of the pubic symphysis (BD). The pubic symphyseal length was the greatest in white South Africans and in males. The pubic symphysis length was the smallest in black females, resulting in a smaller pelvic cavity anteriorly than in white females. Statistically significant variations existed between populations and between sexes in black South Africans but not between white South Africans.

Aging had an effect especially on the pelvic transverse diameters of males and even more so in white South African males presenting with older average ages than black South African males. 
White South African males showed a statistically significant correlation when comparing age and pelvic inlet transverse diameter $\left(\mathrm{C}_{\mathrm{L}} \mathrm{C}_{\mathrm{R}}\right)(\mathrm{p}=0.0031)$ and length of the ischiopubic ramus $\left(\mathrm{DE}_{\mathrm{L}}\right)(\mathrm{p}=0.0433)$.

Transverse pelvic dimensions in females seemed to have been more sensitive to stature variation than in males. Black South African females showed a 5\% statistical significance when comparing height to the pelvic inlet transverse diameter $\left(C_{L} C_{R}\right)(p=0.0463)$ and to the intertuberous diameter $\left(E_{L} E_{R}\right)(p=0.0172)$. White South African females showed a 5\% statistical significance when comparing height to the length of the pubic symphysis (BD) (p= 0.0330), ischiopubic ramus length $\left(\mathrm{DE}_{\mathrm{L}}\right)(\mathrm{p}=0.0066)$ and to the pelvic outlet intertuberous diameter $\left(E_{L} E_{R}\right)(p=0.0191)$. White South Africa males showed a 5\% significance when comparing height and the pelvic inlet anteroposterior diameter $(A B)(p=0.0453)$.

\section{Discussion}

The smaller pelvic inlet diameters in black South African females, could be associated with an inability of the foetal head to engage or the overriding of the vertex during parturition (Standring, 2008). The length of the pubic symphysis in black South African females was also the shortest of all groups considered, rendering the pelvic cavity shallower and smaller anteriorly. Sexual dimorphism in the pelvic inlet may be due to the later maturation of the pubis in females, prolonging the period of growth (Leong, 2006). During the accelerated growth period in adolescence, some individuals are vulnerable to poor socio-economic conditions and food deprivation so that growth in for instance the pubic symphysis is limited. These socio-economic factors could be contributing factors for stunted growth in the pubic symphysis in black South Africans females (Bernard, 1952). In cases where the height of the individual is also affected, the pelvic inlet transverse diameter $\left(C_{L} C_{R}\right)$ may be diminished as well. It might therefore be of value to take the height in black females into account before contemplating normal vaginal delivery.

As the interspinous distance is normally the narrowest part of the pelvic canal through which the foetal head must pass during birth, diameters less than $100 \mathrm{~mm}$ could be a risk for midpelvic arrest (Keller et al., 2003; Moore et al., 2013). As the interspinous distance was sometimes smaller than $100 \mathrm{~mm}$ in black females and stature dependent on a $10 \%$ level of significance $(\mathrm{p}=$ 0.0525), it might also be of value in this regard to take the height of the mother concerned into account when planning assistance during childbirth. 
On the other hand, Kurki (2013) relates population specific variations in the pelvic canal to genetic reasons. Kurki's (2013) findings on an archaeological collection were in line with those we report here. South African females displayed small pelvic inlets relative to a larger lower canal in anteroposterior diameters. Cephalopelvic disproportion might be negated if population specific variations in foetal head size and shape is contemplated to be accommodated by the population specific pelvic canal size and shape. Further research in this regard is necessary to improve understanding regarding the relationship between the shape and size of the foetal head and pelvis.

The greater pelvic transverse inlet diameters noted in white South African males vs. black South African males may have been influenced by aging (Tague, 1994). Pelvic surgery may be technically more challenging in black South Africans, especially in males, because of restricted pelvic access. In addition the smaller subpubic angle in black South African males might contribute to difficult perineal access. Females in general presented with shorter pubic symphyseal lengths and greater pelvic diameters compared to their male counterparts, which might also facilitate intrapelvic and perineal procedures.

\section{Acknowledgements}

We would like to thank Marinda Pretorius and Gerda Venter for their assistance with the photographs, figures and artwork. Also, we thank the anatomy departments of both the University of Pretoria and Sefako Makgatho Health Sciences University for the use of their cadaver material.

\section{References}

Adadevoh, S.W., Hobbs, C., Elkins, T.E., 1989. The relation of the true conjugate to maternal height and obstetric performance in Ghanaians. Int. J. Gynecol. Obst. 28, 243-251.

Bernard, R.M., 1952. The shape and size of the female pelvis. Edinburgh Med. J. 59, 1-15.

Bidmos, M.A., Manger, P.R., 2012. New soft tissue correction factors for stature estimation: results from magnetic resonance imaging. Foren. Sci. Int. 214, 212.

Dayal, M. R., Steyn, M,. 2008. Stature estimation from bones of South African whites. SA. J. Sci. 104, 124-128. 
Frudinger, A., Halligan, S., 2002. Influence of the subpubic arch angle on anal sphincter trauma and anal incontinence following childbirth. Int. J. Obstet Gynaecol. 109, 1207-1212.

Hammer, D., 2001. PAST-PAlaeontological STatistics, ver. 1.89. Palaeontologia electronica 4, $1-9$.

Hong, S. K., Chang, I. H., 2007. Impact of variations in bony pelvic dimensions on performing radical retropubic prostatectomy. J. Urolo. 69, 907-911.

İşcan, M. Y., 2005. Forensic anthropology of sex and body size. Foren. Sci. Int. 147, 107-112.

İşcan, M. Y., Steyn, M., 2013. The human skeleton in forensic medicine. Third edition.

Keller, T. M., Rake, A., 2003. Obstetric MR Pelvimetry: Reference Values and Evaluation of Inter-and Intraobserver Error and Intraindividual Variability 1. Radiol. 227, 37-43.

Killeen, T., Banerjee, S., 2010. Magnetic resonance (MR) pelvimetry as a predictor of difficulty in laparoscopic operations for rectal cancer. J. Surgic. Endosc. 24, 2974-2979.

Kurki, H. K., 2013. Bony pelvic canal size and shape in relation to body proportionality in humans. Am. J. Phys. Anthropol. 151, 88-101.

Leong, A., 2006. Sexual dimorphism of the pelvic architecture: a struggling response to destructive and parsimonious forces by natural \& mate selection. McGill J. Med. 9, 61.

Loth, S. R., Henneberg, M., 1996. Mandibular ramus flexure: a new morphologic indicator of sexual dimorphism in the human skeleton. Am. J. Phys. Anthropol. 99, 473-485.

Lundy, J., Feldesman, M., 1987. Revised equations for estimating living stature from the long bones of the South-African Negro, Bureau Scientific Publ, SA. 83, 54-55.

Merchant, K. M, Villar, J., 2001. Maternal height and newborn size relative to risk of intrapartum caesarean delivery and perinatal distress. Int. J. Obstet. Gynaecol. 108, 689-696.

Moore-Jansen, P. H., Jantz, R. L. 1994. Data collection procedures for forensic skeletal material. University of Tennessee.

Moore, K. L., Dalley, A. F., 2013. Clinically Oriented Anatomy.

Patriquin, M., Loth, S,. 2003. Sexually dimorphic pelvic morphology in South African whites and blacks. J. Compara. Hum. Bio. 53, 255-262.

Patriquin, M., Steyn, M,. 2002. Metric assessment of race from the pelvis in South Africans. Foren. Sci. Inter. 127, 104-113.

Patriquin, M., Steyn, M,. 2005. Metric analysis of sex differences in South African black and white pelves. Foren. Sci. Inter. 147, 119-127. 
Salerno, G., Daniels, I., 2007. Variations in pelvic dimensions do not predict the risk of circumferential resection margin (CRM) involvement in rectal cancer. World J Surg. 31 13151322.

Standring, S., 2008. Gray's Anatomy. The anatomical basis of clinical practice, expert consult, Aubrey Durkin.

Stewart, K., Cowan, D., 1979. Pelvic dimensions and the outcome of trial labour in Shona and Zulu primigravidas. SA Med. J. 55, 847-851.

Tague, R. G., 1994. Maternal mortality or prolonged growth: age at death and pelvic size in three prehistoric Amerindian populations. Am. J. Phys. Anthropol. 95, 27-40. 